\title{
Code Inverse Filtering for Complete Sidelobe Removal in Binary Phase Coded Pulse Compression Systems
}

\author{
Robert C. Daniels and Vilhelm Gregers-Hansen \\ Radar Division, Naval Research Laboratory \\ Washington, DC 20375
}

Key Words: radar signals, pulse compression, binary codes, range sidelobes, inverse filtering

\begin{abstract}
Pulse compression is used in radar systems to improve range resolution while maintaining a high duty cycle. In addition to practical implementation constraints, the key issues for the selection of a pulse-compression waveform are mismatch loss, peak / integrated range sidelobes, and Doppler tolerance. While much progress has been made in the design of nonlinear frequency modulated (FM) chirp waveforms satisfying these requirements, the corresponding performance for binary phase-coded waveforms is often inadequate. In order to improve the range sidelobes achieved with phase-coded waveforms, specially designed mismatched pulse compression filters can be used. Several such approaches have been described in the literature since 1959. This paper will review these techniques and highlight a particular approach using infinite impulse response (IIR) filters, which has received little attention in the past. Using this technique the performance for a number of binary phase codes of different length have been determined and their Doppler tolerance is investigated.
\end{abstract}

\section{INTRODUCTION}

The range sidelobes achieved with matched filter phasecoded pulse compression waveforms, often fail to meet system requirements. This is in contrast to non-linear FM chirp waveforms where excellent performance usually can be realized, even for modest time-bandwidth products [1]. For binary phase codes, the best peak sidelobe performance is obtained with the so-called Barker codes, which for code length up to $\mathrm{N}=13$ will achieve peak sidelobes at a relative level of $20 \cdot \log _{10}(N)$. For longer phase codes, however, range sidelobes rapidly approach a level of the order of only $10 \cdot \log _{10}(N)$. A graph of best known sidelobe levels for matched filtered binary phase code waveforms is shown in Figure 1, along with similar results for best known maximum length shift register codes [2]. The curve $10 \cdot \log _{10}(N)$ is included for comparison.

Many approaches can be used to modify the matched filter design in order to reduce the range sidelobes. These approached usually result in an increased mismatch loss and a potential increase in Doppler sensitivity of the range sidelobes. The best-known example of this approach is the Hamming weighted linear Chirp signal.

This paper will review different approaches proposed in the past for reducing the range sidelobes for phase coded waveforms and, in particular, highlight the approach described by Erikmats [3], which appears to have received little attention since it was originally published. New results are obtained for the performance when this technique is used for longer phase codes. While low sidelobes can be achieved in almost all of these cases, the resulting mismatch loss varies from a modest $0.2 \mathrm{~dB}$ in one particular case to as much as $5-10 \mathrm{~dB}$ or more in other cases.

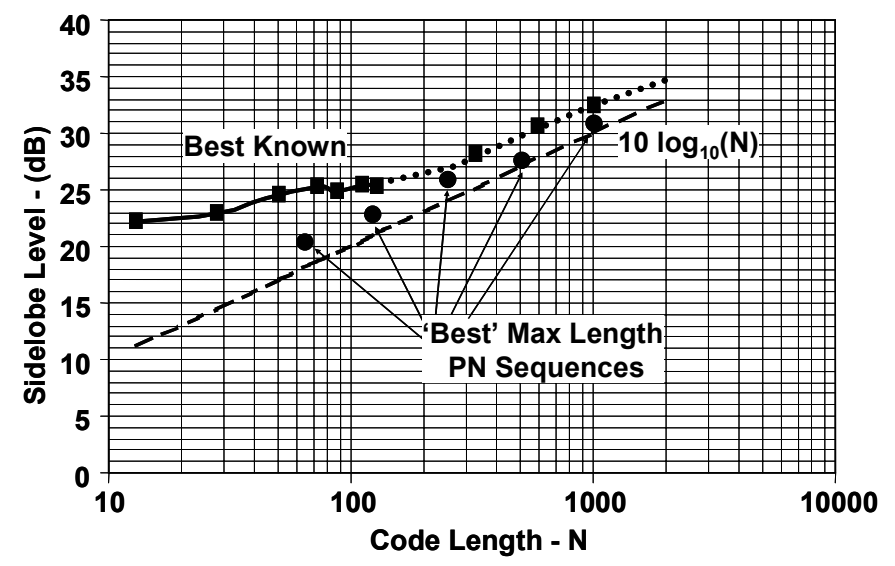

Figure 1 - Matched filter sidelobes for some binary codes.

\section{BINARY PHASE CODES AND SIDELOBE SUPPRESSION}

Binary phase coded waveforms are constructed from a specific binary code of 1 's and -1 's of length $\mathrm{N}$. A basis sub pulse is chosen (often the rectangular function) and this subpulse is repeated $\mathrm{N}$ times, but with a sign determined by the codeword values, to generate the radar waveform at baseband. As described above this would lead to a transmitted waveform where the code elements are modulated in phase by 0 or 180 deg. It is also possible to define complex baseband modulation schemes based on the binary codeword [4].

Some binary codes result in waveforms with better matched filter range sidelobes than others. Much work has been done to find codes with the best possible autocorrelation properties. Barker codes, with an absolute sidelobe level less than or equal to 1 , only exist for length up to $\mathrm{N}=13$. For longer code lengths, exhaustive or near-exhaustive searches have determined the best code words, in terms of range sidelobes, for lengths up to 70 [5].

Methods for reducing the range sidelobes of binary phase 
coded signals have been the subject of much research. Key et al [6] used a time domain approach to sum delayed and scaled versions of the Matched filter output to reduce sidelobes. Mosca [7] and Rihaczek \& Golden [8] used a Fourier series approximation of the inverse of the frequency contribution from the sidelobes. Later, Hua \& Oksman [9] improved on Rihaczek \& Golden by reducing the filter complexity.

A unique approach using complementary codes was developed by Golay [10]. With this approach range sidelobes can be completely eliminated, with no penalty in mismatch loss, but the need to transmit the two complementary codes sequentially (e.g. pulse-to-pulse) makes this approach incompatible with many other radar requirements and Doppler sensitivity could be prohibitive.

The basis for the approach proposed in [3] can be found in the work of Ackroyd \& Ghani [11]. In Section 3 the sidelobe suppression concept of code inverse filtering developed by Erikmats [3] will first be introduced. Section 4 will describe the method of time reversing the data stream in order to implement a recursive filter, which would otherwise be unrealizable. Section 5 presents mismatch loss comparisons for this and other methods; Doppler sensitivity is addressed in Section 6, and implementation considerations are found in Section 7.

\section{CODE INVERSE FILTERING}

The method of code inverse filtering is illustrated in the diagram shown in Figure 2. As usual in the processing of phase-coded waveforms, the first step is matched filtering of the sub-pulse. The output of this sub-pulse matched filter is then sampled in an analog-to-digital converter (ADC) so that the received signal will be a series of $+1 \mathrm{~s}$ and $-1 \mathrm{~s}$ scaled by a common (complex) constant. This signal is then processed through a combined pulse compression and sidelobe removal filter such that its output, for the specific digital binary phase code, is a unit impulse.

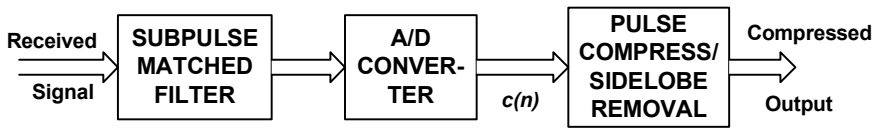

Figure 2 - Code Inverse Filtering Receiver

The following discussion starts with the ideal representation of the signal at the output of the ADC. Since we transmit a binary phase coded signal with sub-pulses modulated by +1 or -1 , we can represent the received digital signal as

$$
c(n)=\sum_{k=0}^{N-1} \delta(n-k) c_{k} \quad c_{k} \in\{-1,1\}
$$

for a codeword of length $N$. If we take the Z-transform equivalent of the signal we find that

$$
C(z)=c_{0}+c_{1} z^{-1}+\ldots+c_{N-1} z^{-N+1} .
$$

If it were possible to design the exact inverse of the codegenerating filter given by equation (2), as the pulse compression/sidelobe removal filter, the output in Figure 2 would be an impulse with zero sidelobes. However, as shown by Ackroyd [12] all polynomials with $+1 /-1$ coefficients, have at least one zero on, or outside, the unit circle. This, in turn, means that a stable response for the inverse of $C(z)$, i.e. $C^{-1}(z)$ is not possible. However, in the case

where none of the zeros fall exactly on the unit circle, it is possible to decompose the polynomial into two parts based on the magnitude of its complex zeros i.e.:

$$
\begin{aligned}
C(z) & =P(z) \cdot Q(z) \\
& \text { where } \\
P(z) & =p_{0}+p_{1} z^{-1}+\ldots+p_{L-1} z^{-L+1} \\
Q(z) & =q_{0}+q_{1} z^{-1}+\ldots+q_{M-1} z^{-M+1}
\end{aligned}
$$

and $N=L+M-1$. Here $P(z)$ is the polynomial factor for all zeros inside the unit circle and $Q(z)$ is the polynomial factor for the zeros outside the unit circle. Thus the IIR filter $P^{-1}(z)$ will be stable and, when used as the first step in the processing of Figure 2, it will remove the sidelobe contribution from zeros inside the unit circle. Finding a way to process the signal through $Q^{-1}(z)$ such that the response is stable, was the key development of the paper by Erikmats [3]. Earlier, in order to circumvent this problem, Ackroyd \& Ghani [11]approximated the ideal response of the sidelobe removal filter using a least squares non-recursive filter implemented as a finite impulse response (FIR) filter. The longer the FIR filter, the closer the sidelobes approach zero. Zoraster continued on Ackroyd \& Ghani's research by using a linear programming nonrecursive filter to approximate the perfect sidelobe removal filter [13].

\section{ERIKMATS' TIME REVERSAL FILTER}

A practical method for processing the signal, such that the sidelobes due to zeros outside the unit circle can be completely removed, was developed in [3]. We show now how time reversal can be used to create the effect of $Q^{-1}(z)$ while still providing a stable response. After the received signal $c(n)$ has been processed through $\boldsymbol{P}^{-1}(z)$ its Ztransform will be given by $Q(z)$ as defined in equation (3). Time reversal changes this Z-transform to:

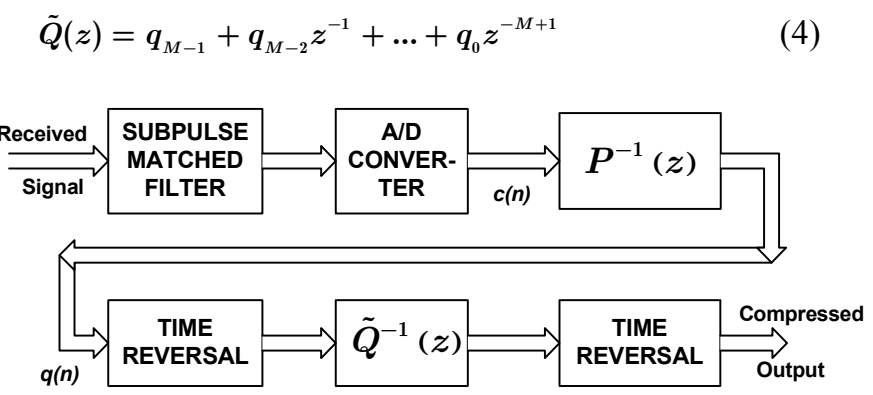

Figure 3 - Erikmats' Code Inverse Sidelobe Removal IIR Filter

The zeros of $\tilde{Q}(z)$ are now the reciprocal of the zeros of $Q(z)$ and they are therefore all located inside the unit circle.

The zeros of the time-reversed data can now be removed 
by a second IIR filter defined by $\tilde{Q}^{-1}(z)$ and when the resulting output is again time-reversed, we will have removed the sidelobes due to the zeros outside the unit circle using only stable IIR filters. Figure 3 depicts the code inverse processing required to completely remove sidelobe levels in a subpulse matched binary phase coded pulse compression.

Table 1 - Comparison of sidelobe reduction filters for N=13 Barker code.

\begin{tabular}{|c|c|c|c|c|}
\hline APPROACH & MF & $\begin{array}{c}\text { SL } \\
\text { FILTER }\end{array}$ & $\begin{array}{c}\text { PSL } \\
(\mathbf{d B})\end{array}$ & $\begin{array}{c}\text { Mismatch } \\
\text { Loss (dB) }\end{array}$ \\
\hline $\begin{array}{c}\text { Key, Fowle, } \\
\text { and Haggarty }\end{array}$ & $\begin{array}{c}F I R \\
N=13\end{array}$ & $\begin{array}{c}F I R \\
N=49\end{array}$ & 45.55 & 0.2133 \\
\hline $\begin{array}{c}\text { Rihaczek and } \\
\text { Golden }\end{array}$ & $\begin{array}{c}F I R \\
N=13\end{array}$ & $\begin{array}{c}F I R \\
N=49\end{array}$ & 45.14 & 0.2141 \\
\hline Erikmats & In IIR & $\begin{array}{c}I I R \\
N P=12\end{array}$ & $>100$ & 0.2137 \\
\hline
\end{tabular}

\section{CODEWORD ANALYSIS AND FILTER COMPARISONS}

A comparison of several of the approaches discussed above, for sidelobe reduction of binary phase-coded waveforms, is shown in Table 1 for the specific case of a Barker code of length $\mathrm{N}=13$. Peak sidelobe level (PSL) and Mismatch Loss are shown for designs, which in a time-domain implementation would require about the same number of multiplies. The matched filter only requires integer coefficients and would add little to complexity. For the two FIR sidelobe reduction filters the number of unique coefficients is actually only 13 due to symmetry and interspersed zeros. This is about the same as required for the feedback coefficients of a IIR filter with $\mathrm{NP}=12$ poles. For the two FIR sidelobe reduction filters, sidelobes better than $45 \mathrm{~dB}$ down are achieved. Note that the PSL achieved with the IIR filtering approach is a theoretical number, which would be subject to practical implementation constraints. The PSL numbers of around 45 $\mathrm{dB}$ would meet system requirements in many applications. Mismatch losses are virtually identical between the three approaches. Generalizing from the results of Table 1 it is concluded that the actual technique used for the design of a sidelobe reduction filter has little effect on Mismatch Loss and hardware implementation considerations vs. achieved sidelobe level would be the main consideration.

Because Erikmats' recursive sidelobe removal filter is easily adapted to different binary codes, it was used to find the smallest possible mismatch loss through an exhaustive computer search of all codes up to length 25 . Table 2 shows the lowest mismatch loss binary phase code found for each code length. In this table it is important to note that there exist equivalent codes with identical mismatch loss properties. Obviously, any code whose Z-transform polynomial has the same roots will generate the same results.

Minimum peak sidelobe codes (MPS) [2] have the property that their autocorrelation function has the lowest peak sidelobe level for a given length code. The mismatch loss of these codes when used with the code inverse sidelobe removal filter has been analyzed for lengths up to 48 as shown in Table 3.
Table 2 - Codes with Lowest Mismatch Loss Using Code Inverse Sidelobe Removal Filter.

\begin{tabular}{|c|c|c|}
\hline $\begin{array}{c}\text { Code } \\
\text { Length }\end{array}$ & Code Word & $\begin{array}{l}\text { Loss } \\
\text { (dB) }\end{array}$ \\
\hline 5 & -+--- & 0.63 \\
\hline 6 & -+---- & 1.70 \\
\hline 7 & +-++--- & 1.52 \\
\hline 8 & -+-++--- & 1.21 \\
\hline 9 & --++-+--- & 2.09 \\
\hline 10 & --++-+---- & 1.69 \\
\hline 11 & +-+--++---- & 0.94 \\
\hline 12 & -+-+--++---- & 0.69 \\
\hline 13 & -+-+--++----- & 0.21 \\
\hline 14 & -+-+--++------ & 0.78 \\
\hline 15 & $\begin{array}{l}+-++-+++-+++-- \\
-\end{array}$ & 0.60 \\
\hline 16 & $\begin{array}{l}-+-++-+++-+++- \\
--\end{array}$ & 1.10 \\
\hline 17 & $\begin{array}{l}+--+-+---++--- \\
---\end{array}$ & 1.25 \\
\hline 18 & $\begin{array}{l}++-+--+-+++--- \\
+---\end{array}$ & 1.01 \\
\hline 19 & $\begin{array}{l}+-++-++-+++-++ \\
+----\end{array}$ & 1.03 \\
\hline 20 & $\begin{array}{l}+--+++--+-+++- \\
+-----\end{array}$ & 0.77 \\
\hline 21 & $\begin{array}{l}-++-+-++-+++-+ \\
++++---\end{array}$ & 0.78 \\
\hline 22 & $\begin{array}{l}---+++--+-+-++ \\
--+-----\end{array}$ & 0.93 \\
\hline 23 & $\begin{array}{l}+-+-+-++-++--+ \\
++-------\end{array}$ & 0.84 \\
\hline 24 & $\begin{array}{l}+-++-+---++++- \\
--+---+---\end{array}$ & 0.79 \\
\hline 25 & $\begin{array}{l}+-++-++-+-+--- \\
-----+++---\end{array}$ & 0.69 \\
\hline
\end{tabular}

\begin{tabular}{|c|c|c|c|}
\hline \multicolumn{4}{|c|}{$\begin{array}{c}\text { Table } 3 \text { - Mismatch Loss of MPS Codes Used With } \\
\text { Code Inverse Sidelobe Removal Filter }\end{array}$} \\
\hline $\begin{array}{l}\text { MPS Code } \\
\text { Length }\end{array}$ & $\begin{array}{l}\text { Mismatch } \\
\text { Loss (dB) }\end{array}$ & $\begin{array}{l}\text { MPS Code } \\
\text { Length }\end{array}$ & $\begin{array}{l}\text { Mismatch } \\
\text { Loss (dB) }\end{array}$ \\
\hline 7 & 1.5192 & 28 & 1.3007 \\
\hline 8 & 10.4498 & 29 & 0.6929 \\
\hline 9 & 3.1694 & 30 & 0.8090 \\
\hline 10 & 1.7693 & 31 & 0.9284 \\
\hline 11 & 1.4845 & 32 & 1.1099 \\
\hline 12 & 0.8148 & 33 & 0.8097 \\
\hline 13 & 0.2137 & 34 & 0.6216 \\
\hline 14 & 4.1539 & 35 & 0.8846 \\
\hline 15 & 1.4659 & 36 & 1.6869 \\
\hline 16 & 4.8594 & 37 & 5.5664 \\
\hline 17 & 1.7607 & 38 & 0.6816 \\
\hline 18 & 24.0035 & 39 & 2.1392 \\
\hline 19 & 2.1788 & 40 & 1.0368 \\
\hline 20 & 1.9333 & 41 & 0.8833 \\
\hline 21 & 0.7832 & 42 & 0.7093 \\
\hline 22 & 1.0772 & 43 & 0.7845 \\
\hline 23 & 1.4168 & 44 & 1.3659 \\
\hline 24 & 0.8093 & 45 & 1.3235 \\
\hline 25 & 0.7101 & 46 & 3.3037 \\
\hline 26 & 3.8237 & 47 & 1.0080 \\
\hline 27 & 0.6471 & 48 & 3.1678 \\
\hline
\end{tabular}




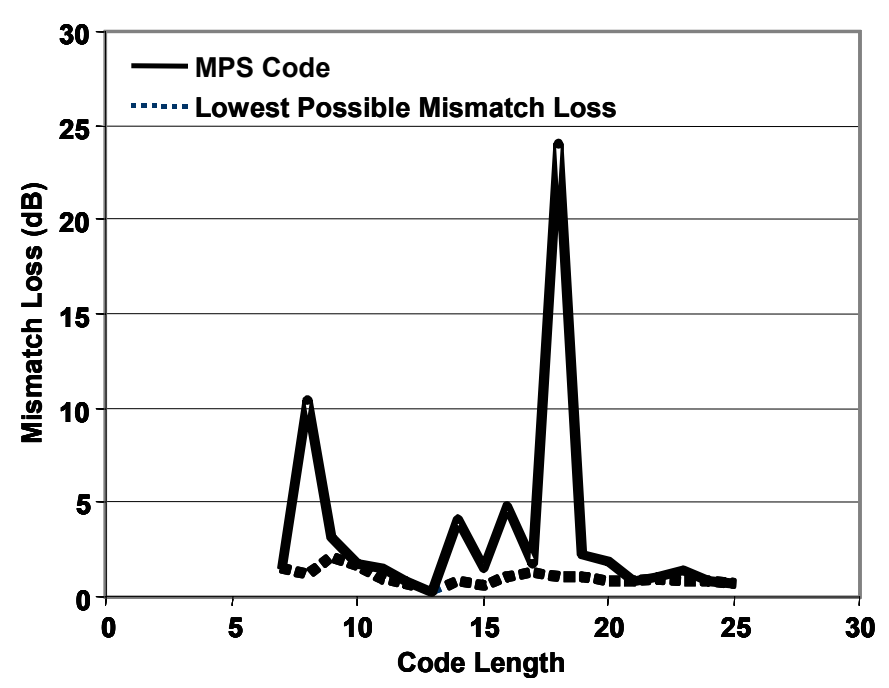

Figure 4 - Mismatch Loss loss of MPS codes and the lowest possible loss, when code inverse filtering is used.

A comparison of the results from the MPS codes with the results from the exhaustive search in Table 2, shows large differences in the mismatch loss resulting from the use of the code inverse filter. MPS codes rarely give the lowest mismatch loss in the sidelobe removal filter as noted in the corresponding graphical comparison of Figure 4. Since we expect the code inverse mismatched filter (as well as all other sidelobe reduction approaches) to perform poorly when one or more zeros of the codeword generating polynomial falls close to the unit circle, the relationship between mismatch loss and the zero closest to the unit circle was explored for the specific case $\mathrm{N}=12$ as shown in Figure 5. While there is no one-to-one correspondence in this graph, it clearly shows a strong correlation.

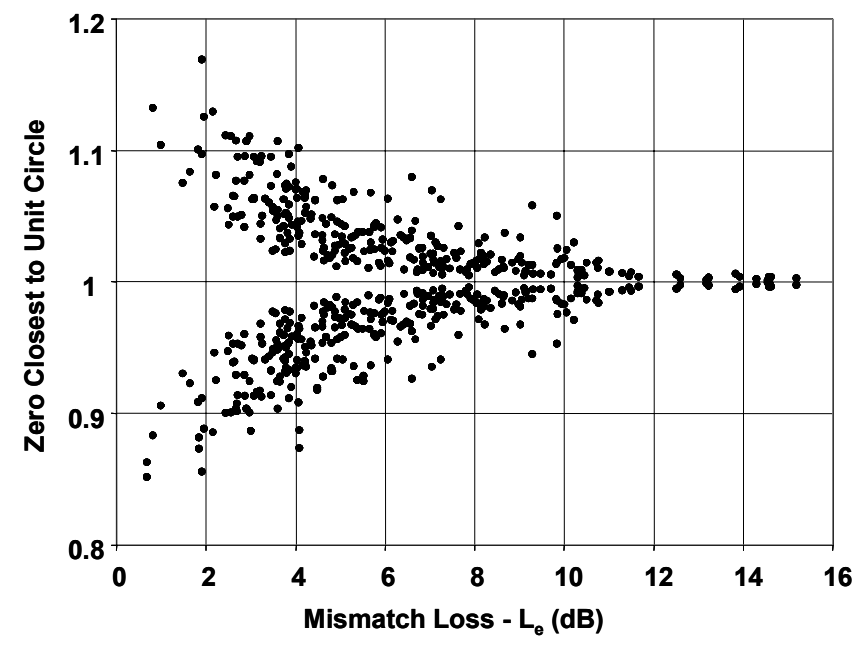

Figure 5 - Scatter plot of zero closest to unit circle versus mismatch loss for $\mathrm{N}=12$ binary codes.

The code inverse approach to sidelobe elimination is not without some disadvantages. Because it is recursive, the filter will have a long impulse response, which adds to the length of the data window which must be kept after processing through the first IIR filter $\left(P^{-1}(z)\right.$ in Figure 3). Also, the processed data needs to be time reversed for the second pass, requiring memory and re-indexing. It is also important to note that, although this approach will work for zeros both inside and outside the unit circle, it will not work for zeros very close to or on the unit circle where the processing gain would become large or infinite. Another limitation in these simulations is the root finding algorithm needed to find the filter coefficients. In MATLAB codes longer than 50 could not be processed by the standard root finding algorithm.

One method, suggested in [14], for creating longer codes is to concatenate codes. Using this method the length of the new code, derived from the two concatenated codes, is the product of the length of the individual codes. Each bit in the new code comes from its respective product with each of the concatenated code bits. The concatenated codes can be processed through cascaded filters in such a way that the mismatch loss simply is the sum of the mismatch losses of the individual codes. Thus if we concatenate two Barker 13 codes we can create a code of length 169 with a mismatch loss of only $2 \cdot(0.2137 \mathrm{~dB})=0.4274 \mathrm{~dB}$

\section{DOPPLER TOLERANCE}

As might be expected, a Doppler shifted return will not achieve the low range sidelobes described above. The

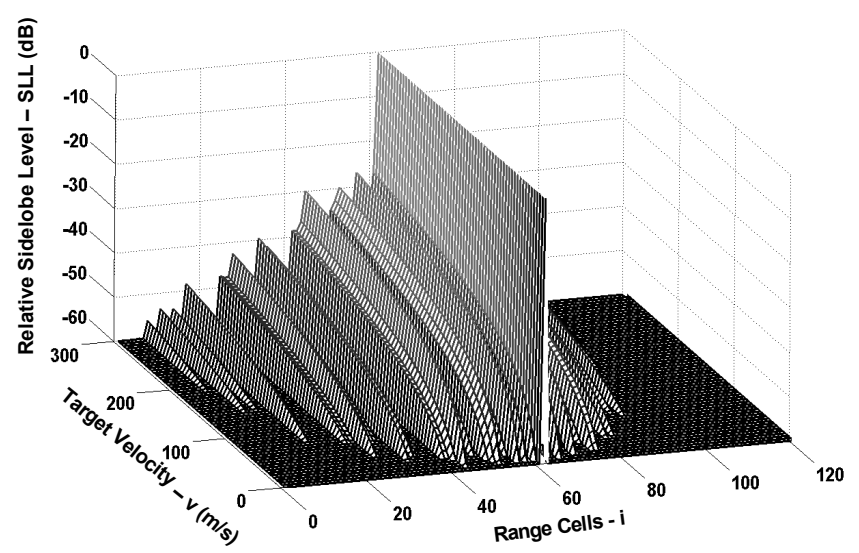

Figure 6 - Effect of Doppler shift on range sidelobes for a $\mathrm{N}=21$ code of duration $\mathrm{T}=10.5 \mu \mathrm{s}$ at $\mathrm{S}$-band $(3.3 \mathrm{GHz})$.

tolerance of the sidelobe level to Doppler shifted returns depends on the radar frequency, the duration of the uncompressed pulse, and of course the actual Doppler shift. As an example, the response of the code inverse pulse compression filter to Doppler shifted inputs, is shown in Figure 6 for a hypothetical S-band radar at $3.0 \mathrm{GHz}$ using an uncompressed pulse width of $10.5 \mu$ s modulated by the binary code of length 21, which was listed in Table 2. The range of radial velocities corresponds to target velocities of 0 to 583 knots. While the degradation is substantial it will be noted that range sidelobes remain close to $30 \mathrm{~dB}$ even for the fastest target. This should be compared to the matched filter sidelobe level of the best binary code of this length of only $20.4 \mathrm{~dB}$. 


\section{IMPLEMENTATION}

The code inverse filters proposed in [3] must be implemented as digital IIR filters of total length equal to the length of the code. A discussion of the design of such IIR filters can be found in [15] and [16, Figure 15.23]. The hardware complexity of such an IIR filter would by similar to that of a FIR filter of the same length. Thus for the comparison presented in Table 1 either the FIR or the IIR approaches to range sidelobe suppression could be implemented with similar hardware complexity. In either case filters with only real coefficients would have to be implemented identically in the in-phase and quadrature channels. However, for better PSL the FIR approaches would require longer filters.

For long phase codes, use of the Fast Fourier Transform (FFT) algorithm is the preferred approach for implementing digital pulse compression. In this case the IIR implementation would not be possible and only a FIR sidelobe reduction filter design would be applicable.

\section{CONCLUSIONS}

This paper has reviewed several techniques for reducing the range sidelobe levels, when binary phase coded pulse compression is used in radar systems. Except for the special case of complementary codes, these approaches all result in a certain mismatch loss, display some degree of Doppler sensitivity, and differ in their specific hardware implementation. All are based on somewhat different mathematical criteria but they all have in common, that the pulse compression operation is implemented using a cascade of a single pulse matched filter and a codeword mismatched filter. The particular approach, where the codeword mismatched filter is implemented as an exact inverse to the code word, was examined in detail. This filter can be implemented as two cascaded IIR filters, of total length equal to the codeword length, but with an intermediate step of time-reversal of the data stream. As long as the code-generating polynomial has no zeroes on, or close to, the unit circle, these filters can be implemented at the cost of a modest mismatch loss, while range sidelobes are completely removed, at least theoretically. Typical mismatch losses are less than $1 \mathrm{~dB}$ and thus performance compares favorably with the frequently used Hamming weighted linear chirp waveform. In a specific example for an S-band radar, it was shown that Doppler sensitivity may well be acceptable and this technique therefore offers an attractive pulse compression approach for systems with very low pulse compression ratios $(<20)$ while requiring good range sidelobes performance.

\section{ACKNOWLEGDEMENT}

The authors wish to acknowledge the support and advice of William W. Shrader of SHRADER Associates, who pointed out the potential benefits of this approach and provided his own unpublished analysis in support of our work.

\section{REFERENCES}

1. V.Gregers-Hansen, "Practical design of a low sidelobe chirp pulse compression system", International Radar Symposium, IRS 98, Munich, Germany, September 15, 1998.

2. F.E.Nathanson, J.P.Reilly, and M.Cohen. Radar Design Principles, 2 ed., New York: McGraw-Hill, 1991.

3. O.Erikmats, "Range sidelobe elimination for discretecoded pulse compression systems", International Conference on Radar, pp. 187-193, Paris, France, December, 1978.

4. J.W.Taylor and H.J.Blinchikoff, "Quadriphase code - A radar pulse compression signal with unique characteristics", IEEE Trans.Aerospace and Electronic Systems, Vol. AES-24, pp. 156-169, March 1988.

5. G.E.Coxson and J.Russo, "Efficient exhaustive search for optimal-peak-sidelobe binary codes", 2004 National Radar Conference, Philadelphia, PA, USA, May, 2004.

6. E.L.Key, E.N.Fowle, and R.D.Haggarty, "A method of sidelobe suppression in phase coded pulse compression systems", MIT Lincoln Laboratory, Report No.Tech Report 209, November 1959.

7. E.Mosca, "Sidelobe reduction in phase-coded pulse compression radars", IEEE Trans.Information Theory, Vol. IT-13, pp. 131-133, January 1967.

8. A.Rihaczek and R.Golden, "Range sidelobe suppression for Barker codes", IEEE Trans.Aerospace and Electronic Systems, Vol. AES-7, pp. 1087-1092, November 1971.

9. C.Hua and J.Oksman, "A new algorithm to optimize Barker code sidelobe suppression filters", IEEE Trans.Aerospace and Electronic Systems, Vol. AES-26, pp. 673-677, July 1990.

10. M.J.Golay, "Complementary series", IRE Trans.Information Theory, Vol. IT-7, pp. 82-87, April 1961.

11. M.H.Ackroyd and F.Ghani, "Optimum mismatched filters for sidelobe suppression", IEEE Trans.Aerospace and Electronic Systems, Vol. AES-9, pp. 214-218, March 1973.

12. M.H.Ackroyd, "Synthesis of efficient Huffman sequences", IEEE Trans.Aerospace and Electronic Systems, Vol. AES-8, pp. 2-8, January 1972.

13. S.Zoraster, "Minimum peak range sidelobe filter for binary phase-coded waveforms", IEEE Trans.Aerospace and Electronic Systems, Vol. AES-16, pp. 112-115, January 1980.

14. E.Hollis, "Comparison of combined Barker codes for radar use", IEEE Trans.Aerospace and Electronic Systems, Vol. AES-3, pp. 141-144, January 1967.

15. H.Urkowitz, "Analysis and synthesis of delay line periodic filters", IRE Trans.Circuit Theory, Vol. CT-4, No.2, pp. 41-53, June 1957.

16. W.W.Shrader and V.Gregers-Hansen. "MTI Radar", Ch.15, pp. 15.1-15.72. In Radar Handbook, 2. ed., Editor: M.I.Skolnik. New York: McGraw-Hill, 1990. 


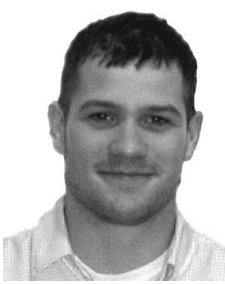

Robert C. Daniels was born in Tunkhannock, PA on August 24th, 1980. He received B.S. degree (with distinction) in Electrical Engineering and Mathematics in 2004 from The Pennsylvania State University. He is currently pursuing an M.S. degree in electrical engineering at The University of Texas at Austin. During the summer of 2004 he was an ONR intern with the Naval Research Laboratory in Washington D.C. At NRL Robert researched methods related to signal processing in pulse compression radar systems. His current research interests are signal processing in radar systems and multiple antenna wireless communication systems.

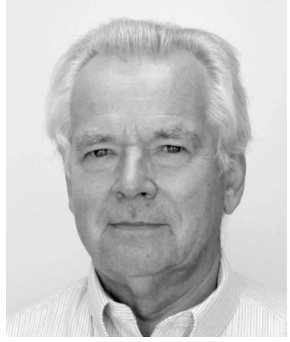

Vilhelm Gregers-Hansen (LF'96) was born in Odense, Denmark in 1934. He received his M.Sc. EE degree from the Technical University of Denmark in 1959 and following service in the Royal Danish Navy he worked as a Scientist at the SHAPE Technical Center in The Hague, Holland during 1960-66. Following 3 years at the Technical University of Denmark, he joined Raytheon Company in Wayland, Massachusetts in 1969 where he remained until his retirement in 1995. At Raytheon he became a Consulting Scientist and was Technical Director for the AN/SPS-49 US Navy radar program for a number of years. In 1995 he joined the Naval Research Laboratory in Washington, DC, where he supports current Navy radar developments and also led the team that developed the $94 \mathrm{GHz}$ WARLOC radar; the worlds highest power radar at W-band. Mr. Gregers-Hansen was elected to the grade of Fellow by the Institute of Electrical and Electronics Engineers in 1980. 